\title{
Cardiac contractile dysfunction in insulin-resistant rats fed a high-fat diet is associated with elevated CD36-mediated fatty acid uptake and esterification
}

\author{
D. M. Ouwens • M. Diamant • M. Fodor • \\ D. D. J. Habets • M. M. A. L. Pelsers • M. El Hasnaoui • \\ Z. C. Dang • C. E. van den Brom • R. Vlasblom • \\ A. Rietdijk • C. Boer • S. L. M. Coort • J. F. C. Glatz • \\ J. J. F. P. Luiken
}

Received: 7 March 2007 / Accepted: 22 May 2007 / Published online: 18 July 2007

(C) Springer-Verlag 2007

\begin{abstract}
Aims/hypothesis Changes in cardiac substrate utilisation leading to altered energy metabolism may underlie the development of diabetic cardiomyopathy. We studied cardiomyocyte substrate uptake and utilisation and the role of the fatty acid translocase CD36 in relation to in vivo cardiac function in rats fed a high-fat diet (HFD).

Methods Rats were exposed to an HFD or a low-fat diet (LFD). In vivo cardiac function was monitored by echocardiography. Substrate uptake and utilisation were determined in isolated cardiomyocytes.
\end{abstract}

Electronic supplementary material The online version of this article (doi:10.1007/s00125-007-0735-8) contains supplementary material, which is available to authorised users.

D. M. Ouwens $(\varangle) \cdot$ C. E. van den Brom •

R. Vlasblom $\cdot$ A. Rietdijk

Department of Molecular Cell Biology, Section of Signal

Transduction and Ageing, Leiden University Medical Centre,

Postzone S1-P, P.O. Box 9600, NL-2300 RC Leiden,

The Netherlands

e-mail: d.m.ouwens@lumc.nl

M. Diamant $\cdot$ Z. C. Dang $\cdot$ C. E. van den Brom •

R. Vlasblom

Department of Endocrinology, Diabetes Centre,

VU University Medical Centre,

Amsterdam, the Netherlands

M. Fodor

Department of Anatomy and Embryology/Central Animal Facility,

Leiden University Medical Centre,

Leiden, the Netherlands
Results Feeding an HFD for 8 weeks induced left ventricular dilation in the systolic phase and decreased fractional shortening and the ejection fraction. Insulin-stimulated glucose uptake and proline-rich Akt substrate 40 phosphorylation were $41 \%(p<0.001)$ and $45 \%(p<0.05)$ lower, respectively, in cardiomyocytes from rats on the HFD. However, long-chain fatty acid (LCFA) uptake was 1.4-fold increased $(p<0.001)$ and LCFA esterification into triacylglycerols and phospholipids was increased 1.4- and 1.5-fold, respectively (both $p<0.05$ ), in cardiomyocytes from HFD compared with LFD hearts. In the presence of the CD36 inhibitor sulfo- $N$-succinimidyloleate, LCFA uptake and esterification were similar in LFD and HFD cardiomyocytes. In HFD hearts CD36 was relocated to the sarcolemma, and basal phosphorylation of a mediator of CD36-trafficking, i.e. protein kinase B (PKB/Akt), was increased.

D. D. J. Habets · M. M. A. L. Pelsers • M. El Hasnaoui •

S. L. M. Coort · J. F. C. Glatz · J. J. F. P. Luiken

Department of Molecular Genetics, Cardiovascular Research

Institute Maastricht, Maastricht University,

Maastricht, the Netherlands

Z. C. Dang • C. E. van den Brom • R. Vlasblom • C. Boer Laboratory for Physiology, VU University Medical Centre, Amsterdam, the Netherlands

J. J. F. P. Luiken

Department of Biochemical Physiology and Institute of Biomembranes, Utrecht University,

Utrecht, the Netherlands 
Conclusions/interpretation Feeding rats an HFD induced cardiac contractile dysfunction, which was accompanied by the relocation of CD36 to the sarcolemma, and elevated basal levels of phosphorylated PKB/Akt. The permanent presence of $\mathrm{CD} 36$ at the sarcolemma resulted in enhanced rates of LCFA uptake and myocardial triacylglycerol accumulation, and may contribute to the development of insulin resistance and diabetic cardiomyopathy.

Keywords CD36 - Contractile dysfunction .

Diabetic cardiomyopathy · Fatty acid utilisation .

Glucose uptake $\cdot$ High-fat feeding $\cdot$ Insulin resistance

$\begin{array}{ll}\text { Abbreviations } \\ \text { ACC } & \text { acetyl-coenzyme A carboxylase } \\ \text { AMPK } & \text { AMP kinase } \\ \text { ED } & \text { end diastole } \\ \text { EDD } & \text { end diastolic diameter } \\ \text { EDV } & \text { end diastolic volume } \\ \text { EF } & \text { ejection fraction } \\ \text { ES } & \text { end systole } \\ \text { ESD } & \text { end systolic diameter } \\ \text { ESV } & \text { end systolic volume } \\ \text { FS } & \text { fractional shortening } \\ \text { HFD } & \text { high-fat diet } \\ \text { IVSWT } & \text { interventricular septum wall thickness } \\ \text { LCFA } & \text { long-chain fatty acid } \\ \text { LFD } & \text { low-fat diet } \\ \text { LV } & \text { left ventricle } \\ \text { PI3K } & \text { phosphatidylinositol } 3 \text { '-kinase } \\ \text { PKB/Akt } & \text { protein kinase B } \\ \text { PPAR } \alpha & \text { peroxisome proliferator activated receptor } \alpha \\ \text { PRAS40 } & \text { proline-rich Akt substrate } 40 \\ \text { PWT } & \text { posterior wall thickness } \\ \text { SSO } & \text { sulfo- } N \text {-succinimidyloleate } \\ \text { VD } & \text { left ventricular diameter }\end{array}$

\section{Introduction}

Diabetic cardiomyopathy (DCM) is a common complication in type 2 diabetes [1]. Changes in myocardial energy metabolism, due to altered substrate handling, characterise diabetes-related heart disease and DCM [1]. Studies in rodent models, including rats fed a high-fat diet (HFD) [2], leptin-deficient and -resistant animals [3-6] and transgenic mice with cardiac-restricted peroxisome proliferator activated receptor $\alpha(\operatorname{PPAR} \alpha)$ overexpression [7], indicate that intramyocardial accumulation of triacylglycerol metabolites (lipotoxicity) and myocardial insulin resistance may underlie diabetes-related cardiac dysfunction $[1,8]$.
Among the putative causes of myocardial triacylglycerol accumulation are elevations in long-chain fatty acid (LCFA) uptake $[6,7,9]$. In the heart, approximately $50 \%$ of LCFA uptake is mediated by the fatty acid translocase CD36 [10, 11]. Under physiological conditions, both insulin and contraction stimulate LCFA uptake by inducing the translocation of CD36 from an intracellular pool to the sarcolemma, illustrating that CD36 is also directly involved in the dynamic utilisation of LCFA by cardiomyocytes. This acute translocation of CD36 involves activation of phosphatidylinositol 3'-kinase (PI3K)/protein kinase B (PKB/Akt)- and AMP-kinase (AMPK)-dependent signalling cascades in response to insulin and contraction, respectively [11].

Interestingly, CD36 deletion rescues the cardiac dysfunction, metabolic abnormalities and myocardial triacylglycerol accumulation observed in transgenic mice with cardiac-restricted PPAR $\alpha$ overexpression [7], suggesting a critical role for CD36 in the development of diabetesrelated heart disease [12]. We hypothesised that long-term alterations in the functional pool of CD36 lead to myocardial accumulation of (toxic) lipid metabolites and insulin resistance and contribute to the development of diabetesrelated heart disease. To examine this, we first measured in vivo cardiac dimensions by echocardiography in rats exposed to a high-fat diet (HFD), which impairs myocardial insulin signalling $[2,13]$. Subsequently, isolated cardiomyocytes were used to determine changes in substrate uptake and LCFA metabolism under basal conditions and after incubations with insulin and with oligomycin; the latter compound induces a contraction-like elevation of intracellular AMP levels and concomitant activation of AMPK [14]. Finally, we assessed whether the observed alterations could be ascribed to $\mathrm{CD} 36$ in hearts from rats on the HFD by analysing the effects of the CD36 inhibitor sulfo- $N$-succinimidyloleate (SSO) on myocardial LCFA uptake and utilisation in isolated cardiomyocytes, and by studying the expression and subcellular localisation of CD36 in cardiac ventricular tissue.

\section{Materials and methods}

Animals The investigation conformed to the Guide for the Care and Use of Laboratory Animals published by the NIH (NIH Publication No. 85-23, revised 1996) and was approved by the VU University Medical Centre Animal Care Committee. Adult male Wistar WU rats $(n=31$; mean body weight $361 \pm 24 \mathrm{~g}$ ) were purchased from Harlan CBP (Horst, the Netherlands), and fed an HFD or an isocaloric low-fat diet (LFD) [2]. At week 8, rats fasted overnight underwent an OGTT, and post-load blood glucose levels were determined 15, 30, 60, 90 and 
$120 \mathrm{~min}$ after ingestion of $2 \mathrm{~g}$ glucose $/ \mathrm{kg}$ body weight [2]. After 10 weeks on the diet, blood was collected for determination of glucose and insulin levels. Rats were then anaesthetised with an intraperitoneal injection of sodium pentobarbital, and hearts were removed for the isolation of cardiomyocytes [15].

Diets Experimental diets were obtained from Hope Farms (Woerden, the Netherlands; HFD, catalogue no. 4148.02; LFD, catalogue no. 4148.01). The LFD consisted of $8 \%$ by weight of total fat, $22 \%$ by weight of protein and $60 \%$ by weight of carbohydrate; the HFD contained $25 \%$ by weight of fat, $32 \%$ by weight of protein and $25 \%$ by weight of carbohydrate, as well as more palmitate $(91.12 \mathrm{~g} / \mathrm{kg})$ and oleate $(100.24 \mathrm{~g} / \mathrm{kg})$ compared with the LFD (29.12 and $32.08 \mathrm{~g} / \mathrm{kg}$, respectively). In the group fed the HFD, 50.4\% of the ingested calories were derived from fat compared with $16.4 \%$ in the group fed the LFD.

In vivo cardiac function Non-invasive transthoracic echocardiograms were recorded at heart rates of 350-400 beats per minute in rats anaesthetised with $1.5 \%$ isoflurane in a mixture of $\mathrm{N}_{2} \mathrm{O} / \mathrm{O}_{2}(1 / 2, \mathrm{vol} / \mathrm{vol})$ [16] before and 8 weeks after initiation of the diet by an Aloka echo machine (ProSound SSD-4000) using a 13-MHz linear interfaced array transducer. $\mathrm{M}$ - and B-mode images were obtained in the parasternal long- and short-axis views of the left ventricle (LV) at mid-papillary level. At end systole (ES) and end diastole (ED), LV lumen diameter (D), LV ventricular diameter (VD) and posterior (PWT) and interventricular septum (IVSWT) wall thicknesses were determined for three cardiac cycles and averaged. The LV dimensions were used to calculate systolic parameters [17]: fractional shortening $(\mathrm{FS} \%)=[(\mathrm{EDD}-\mathrm{ESD}) / \mathrm{EDD})] \times 100 \%$, where $\mathrm{EDD}$ is end diastolic diameter and ESD is end systolic diameter; end diastolic volume $(E D V)=[E D D]^{3}$; end systolic volume $(\mathrm{ESV})=[\mathrm{ESD}]^{3}$; and ejection fraction $(\mathrm{EF} \%)=[(\mathrm{EDV}-$ $\mathrm{ESV}) / \mathrm{EDV}] \times 100 \%$. LV mass $(\mathrm{LVM})$ was determined according to the uncorrected cube assumption: $1.05 \times$ $\left[(\mathrm{EDD}+\mathrm{PWT}+\mathrm{IVS})^{3}-\mathrm{EDV}\right]$, where 1.05 is the specific gravity of the myocardium [18].

Plasma and tissue determinations Blood glucose was measured from tail bleeds using a glucose analyser (HemoCue, Angelholm, Sweden). Plasma insulin was measured using ELISA (EZRMI-13K; Linco Research, St Charles, MO, USA). Myocardial triacylglycerol content was determined in ventricular lysates from a separate set of animals as described in $[2]$.

Treatment of isolated cardiomyocytes Cardiomyocytes were prepared using a Langendorff perfusion system as described in $[15,19]$. Isolated cardiomyocytes were incubated for $30 \mathrm{~min}$ at room temperature with $0.5 \%$ DMSO or SSO [9]. The CD36-specific inhibitor SSO [20] was used to investigate the involvement of CD36 in basal LCFA uptake. SSO was applied at a concentration of $0.4 \mathrm{mmol} / \mathrm{l}$, which effectively inhibits $\operatorname{CD} 36[15,21]$. Then, cells were washed and incubation was continued for $15 \mathrm{~min}$ at $37^{\circ} \mathrm{C}$ with continuous shaking. Thereafter, the DMSO-treated cardiomyocytes were kept for another $15 \mathrm{~min}$ at $37^{\circ} \mathrm{C}$ and either kept untreated (basal) or incubated with insulin or oligomycin. To achieve optimal stimulation of glucose and LCFA uptake [22], insulin and oligomycin were used at a concentration of $10 \mathrm{nmol} / \mathrm{l}$ and $30 \mu \mathrm{mol} / \mathrm{l}$, respectively. At this concentration, the ATPsynthase inhibitor oligomycin induces an increase in the intracellular AMP/ATP ratio, thereby activating AMPKmediated signalling pathways without inhibiting oxygen consumption [14].

Glucose uptake, palmitate uptake and oxidation, and incorporation into intracellular lipid pools Following agonist treatment, $\left[{ }^{3} \mathrm{H}\right]$-2-deoxyglucose and $\left[1-{ }^{14} \mathrm{C}\right]$ palmitate uptake were simultaneously determined during a 3-min incubation [15]. Rates of $\left[1-{ }^{14} \mathrm{C}\right]$ palmitate oxidation (measured as the production of ${ }^{14} \mathrm{CO}_{2}$ ) and esterification (measured as incorporation of the ${ }^{14} \mathrm{C}$ label into intracellular phospholipids [PL], and triacylglycerol) were determined after a 20-min incubation [9].

Western blotting Phosphorylation of proline-rich Aktsubstrate 40 (PRAS40-Thr246) and acetyl-coenzyme A carboxylase-Ser79 (ACC-Ser79) was determined in homogenates of cardiomyocytes by western blotting using phospho-specific antibodies $[9,13]$. PKB/Akt-Ser473 phosphorylation was determined in ventricular homogenates as described in [2]. Expression levels of CD36 were determined by western blotting using the monoclonal CD36 MO25 antibody [23]. Immunoblots were quantified by densitometric analysis of the films [2].

Cardiac morphology and subcellular localisation of CD36 Cardiac ventricular tissue collected in a previous study [2] was routinely embedded in paraffin (Histowax; Leica Microsystems, Wetzlar, Germany). Sections $(5 \mu \mathrm{m})$ were cut, and mounted on slides coated with 3-aminopropyltriethoxisilane (Menzal, Darmstadt, Germany). After deparaffinisation and rehydration, the slides were used for determination of cardiomyocyte cross-sectional area or immunohistochemical staining of CD36.

Cross-sectional area was determined in randomly chosen fields in haematoxylin/eosin-stained slides using NIH image analysis software (National Institutes of Health, Bethesda, MD, USA) for 20-30 cells per heart and normalised to sarcomere length [24]. 
For CD36 localisation, sections were rinsed extensively with Tris-buffered saline (TBS; $50 \mathrm{mmol} / 1$ Tris-Cl, $500 \mathrm{mmol} / \mathrm{l} \mathrm{NaCl}, \mathrm{pH}$ 7.6), and incubated overnight with the monoclonal CD36 MO25 antibody [23] diluted 1:2,000 in TBS containing $0.5 \%$ Triton $\mathrm{X}-100, \mathrm{pH} 7.6$ at $4^{\circ} \mathrm{C}$. After several washes in TBS, sections were incubated for $1.5 \mathrm{~h}$ at room temperature with biotinylated anti-mouse $\operatorname{IgG}$ (Vector Laboratories, Burlingame, CA, USA), diluted 1:250 in TBS, followed by incubation for $1 \mathrm{~h}$ at room temperature with avidin-biotin-peroxidase (1:400; ABC Elite kit; Vector Laboratories). Tissue-bound peroxidase was visualised with the 3,3'-diaminobenzidine tetrahydrochloride (DAB) chromogen reaction $(7.5 \mathrm{mg} \mathrm{DAB}, 5 \mu \mathrm{l}$ $30 \% \mathrm{H}_{2} \mathrm{O}_{2}$ in $15 \mathrm{ml} 50 \mathrm{mmol} / \mathrm{l}$ Tris-Cl, $\mathrm{pH} 7.6$ ) for $10 \mathrm{~min}$ at room temperature. Sections were then rinsed with distilled water, dehydrated in a graded series of ethanol, cleared in xylene, and coverslipped with Entellan (Merck, Darmstadt, Germany). Sections were analysed using a Leica DM-LB light microscope (Leica, Rijswijk, the Netherlands). Digital images were taken at $\times 200$ magnification using a Leica DC500 digital camera, and quantified using Quantity One software (Bio-Rad Laboratories, Veenendaal, the Netherlands).

Statistical analysis Unless indicated otherwise, data are expressed as means $\pm \mathrm{SE}$ for the indicated number of animals ( $n$ ). Statistical analysis was performed in SPSS for Mac OS X version 11.0.4 (SPSS Inc., Chicago, IL, USA). Differences between the LFD and HFD groups were determined using independent $t$ tests. The paired sample $t$ test was used to define the effect of agonist incubations on cardiomyocyte preparations. $p<0.05$ was considered as statistically significant.

\section{Results}

HFD induces cardiac contractile dysfunction Feeding rats isocaloric LFD or HFD diets for 8 weeks induced similar weight gains in the two groups (Table 1), but mild glucose intolerance, as determined by an oGTT, in HFD- vs LFD-rats $(p<0.05$; Fig. 1a). Figure $1 \mathrm{~b}$ shows the LV dimensions at the start and 8 weeks after initiation of the diet interventions. Baseline parameters were similar in the two groups (Table 1). After 8 weeks on the diet, LVM, ED-VD, EDD and EDIVSWT showed comparable increases in LFD and HFD hearts (all $p<0.05$ ), whereas ED-PWT was unaffected by the diet (Table 1). In the ES phase, both ES-PWT and ESIVSWT were increased in LFD hearts only (both $p<0.005$; Table 1). ES-VD was similarly affected by LFD and HFD feeding. ESD was increased in LV from HFD-fed vs LFDfed rats $(p<0.01$; Table 1). FS\% was increased in LFD hearts $(p<0.05)$, and decreased in HFD hearts during the course of
Table 1 In vivo cardiac characteristics of rats before and after 8 weeks on an HFD or a LFD

\begin{tabular}{|c|c|c|c|}
\hline & $\begin{array}{l}\text { Start } \\
(n=24)\end{array}$ & $\begin{array}{l}\text { LFD } \\
(n=8)\end{array}$ & $\begin{array}{l}\text { HFD } \\
(n=16)\end{array}$ \\
\hline \multicolumn{4}{|l|}{ Physiological parameters } \\
\hline Body weight (g) & $295 \pm 4$ & $476 \pm 8^{*}$ & $463 \pm 8^{*}$ \\
\hline Left ventricular mass (mg) & $637 \pm 15$ & $890 \pm 18^{*}$ & $882 \pm 32 *$ \\
\hline \multicolumn{4}{|l|}{ LV diastolic parameters } \\
\hline Posterior wall thickness (mm) & $1.63 \pm 0.03$ & $1.80 \pm 0.07$ & $1.71 \pm 0.05$ \\
\hline Lumen diameter (mm) & $6.92 \pm 0.07$ & $7.55 \pm 0.20^{*}$ & $7.68 \pm 0.10^{*}$ \\
\hline $\begin{array}{l}\text { Interventricular septum } \\
\text { wall thickness }(\mathrm{mm})\end{array}$ & $1.37 \pm 0.03$ & $1.67 \pm 0.08^{*}$ & $1.54 \pm 0.04 *$ \\
\hline Ventricular diameter $(\mathrm{mm})$ & $9.80 \pm 0.06$ & $10.86 \pm 0.13 *$ & $10.89 \pm 0.12 *$ \\
\hline \multicolumn{4}{|l|}{ LV systolic parameters } \\
\hline Posterior wall thickness (mm) & $2.93 \pm 0.06$ & $3.45 \pm 0.12 *$ & $3.00 \pm 0.08^{* *}$ \\
\hline Lumen diameter $(\mathrm{mm})$ & $3.41 \pm 0.10$ & $3.32 \pm 0.25$ & $4.07 \pm 0.12^{* * * *}$ \\
\hline $\begin{array}{l}\text { Interventricular septum } \\
\text { wall thickness (mm) }\end{array}$ & $2.51 \pm 0.05$ & $2.85 \pm 0.09^{*}$ & $2.67 \pm 0.06$ \\
\hline Ventricular diameter (mm) & $8.81 \pm 0.07$ & $9.82 \pm 0.12 *$ & $9.81 \pm 0.10^{*}$ \\
\hline Fractional shortening (\%) & $50.8 \pm 1.3$ & $56.3 \pm 2.4^{*}$ & $47.1 \pm 1.7^{* * * *}$ \\
\hline Ejection fraction (\%) & $87.5 \pm 1.0$ & $91.1 \pm 1.4$ & $84.9 \pm 1.0^{*, * *}$ \\
\hline
\end{tabular}

Data are means \pm SE

$* p<0.05$ vs start

$* * p<0.01$ vs LFD

the diet intervention ( $p<0.02$; Table 1). Accordingly, $\mathrm{EF} \%$ was decreased in HFD compared with LFD hearts $(p<0.05$; Table 1). Collectively, these data show that HFD induced cardiac contractile dysfunction in rats.

Insulin action, but not AMPK signalling, is impaired in HFD cardiomyocytes We used cardiomyocytes to unravel the diet-induced molecular alterations in energy substrate uptake and intracellular signalling pathways. Cardiomyocytes were isolated from a separate group of rats, fed either the LFD or the HFD under identical experimental conditions. At killing, body, heart and liver weights were similar between LFD- and HFD-fed rats, whereas perirenal fat pad weight was $50 \%$ higher in HFD- than in LFD-fed rats $(p<0.001$; Table 2$)$. Furthermore, HFD feeding increased blood glucose levels $(p<0.02)$ and lowered plasma insulin levels $(p<0.05)$.

Basal 2-deoxyglucose glucose uptake rates were $7.3 \pm 0.8$ and $11.0 \pm 1.6 \mathrm{nmol} \mathrm{m^{-1 }}$ [g wet cell mass] ${ }^{-1} \mathrm{C}$ in LFD and HFD cells, respectively ( $p=0.063$; Fig. 2$)$. Insulin stimulated 2-deoxyglucose uptake 3.7-fold in LFD cardiomyocytes and 2.2-fold in HFD cardiomyocytes (both $p<0.001$ vs basal, $p<0.02$ HFD vs LFD), indicating reduced myocardial insulin responsiveness. Oligomycin had similar stimulatory effects on 2-deoxyglucose uptake in HFD and LFD cardiomyocytes (HFD, 1.6-fold stimulation; LFD, 1.7fold stimulation; both $p<0.05$ vs basal).

Activation of phosphatidylinositol 3'-kinase (PI3K)/ protein kinase $\mathrm{B}$ (PKB/Akt)- and AMP-kinase (AMPK)- 


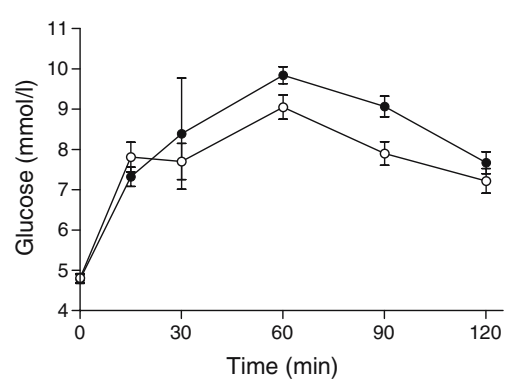

b

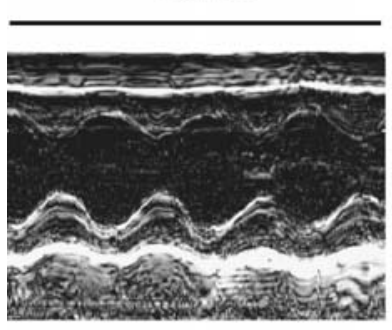

$t=8$ weeks

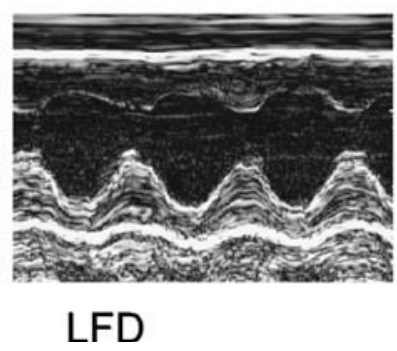

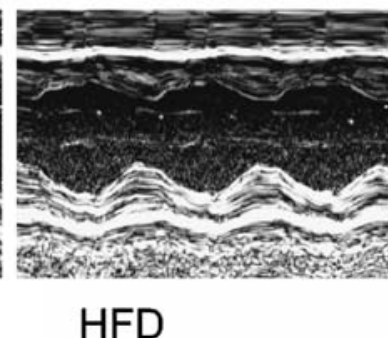

Fig. 1 a Blood glucose levels after an oral glucose load in rats fed the HFD (closed circles; $n=16$ ) and the LFD (open circles; $n=8$ ). Data are means \pm SE. b Representative echocardiographic M-mode images from at least three cardiac contractile cycles at the start of the diet intervention and 8 weeks after initiation of the LFD and HFD, respectively

dependent signalling cascades has been implicated in the stimulation of glucose uptake in response to insulin and oligomycin, respectively. We studied whether changes in the activation of these signalling pathways paralleled the observed alterations in glucose uptake rates in HFD cells. Therefore, phosphorylation of PRAS40-Thr246 and ACCSer79 were examined as respective distal determinants for PKB/Akt and AMPK activity [13, 25], as the presence of bovine serum albumin (mol. mass $66 \mathrm{kDa}$ ) in the cardiomyocyte homogenates interfered with direct measurement of PKB/Akt (mol. mass $64 \mathrm{kDa}$ ) and AMPK $\alpha$ phosphorylation (mol. mass $62 \mathrm{kDa}$ ). Basal PRAS40-Thr246 phosphorylation was 1.7 -fold higher in HFD than in LFD cells $(p<0.05$; Fig. 3a,b). Insulin stimulated PRAS40-Thr246 phosphorylation 12.0-fold in LFD cells $(p<0.001)$ and 4.0fold in HFD cells $(p<0.001)$, thus confirming reduced insulin responsiveness. Oligomycin did not affect PRAS40Thr246 phosphorylation.

ACC-Ser79 phosphorylation was measured as a determinant of AMPK activity [25]. Basal levels of Ser79-

Table 2 Characteristics of rats after 10 weeks on a high- or low-fat diet

\begin{tabular}{lcc}
\hline & $\begin{array}{c}\text { LFD } \\
(n=14)\end{array}$ & $\begin{array}{c}\text { HFD } \\
(n=17)\end{array}$ \\
\hline Body composition & & \\
Body weight at killing (g) & $465 \pm 5$ & $472 \pm 10$ \\
Heart weight (\% body weight) & $0.445 \pm 0.007$ & $0.431 \pm 0.012$ \\
Liver weight (\% body weight) & $3.10 \pm 0.07$ & $3.02 \pm 0.08$ \\
Perirenal fat pad weight & $1.63 \pm 0.08$ & $2.43 \pm 0.14 *$ \\
$\quad(\%$ body weight) & & \\
Plasma characteristics & & \\
Blood glucose (mmol/l) & $5.50 \pm 0.08$ & $5.78 \pm 0.08^{* *}$ \\
Plasma insulin (pmol/1) & $269 \pm 18$ & $216 \pm 16^{* * *}$ \\
\hline Data are means \pm SE & & \\
$* p<0.001$ vs LFD & & \\
$* * p<0.02$ vs LFD & & \\
$* * * p<0.05$ vs LFD & &
\end{tabular}

phosphorylated ACC were similar in LFD and HFD cells (Fig. 3c,d). Insulin had no effect on ACC-Ser79 phosphorylation, whereas oligomycin stimulated ACC-Ser79 phosphorylation in LFD and HFD cells to a similar extent (HFD, 3.0-fold stimulation; LFD, 2.9-fold stimulation; both $p<0.001$ vs basal), indicating that AMPK signalling was not affected by high-fat feeding.

HFD feeding results in increased CD36-mediated LCFA uptake Basal rates of LCFA uptake were increased 1.4-fold $(p<0.001)$ in HFD compared with LFD cells (Fig. 4). The CD36 inhibitor SSO reduced basal LCFA uptake by $44 \%$ $(p<0.001)$ and $23 \%(p<0.05)$ in HFD and LFD cardiomyocytes, respectively. Notably, the absolute rates of LCFA uptake were similar between SSO-treated LFD and HFD cells (Fig. 4), suggesting that the increased basal LCFA uptake rates in HFD cells can be ascribed to changes in the sarcolemmal pool of CD36. Insulin stimulated LCFA uptake 1.3-fold in LFD cells ( $p<0.02$ vs basal) but had no effect in HFD cells $(p<0.02$ vs LFD; Fig. 4). Oligomycin

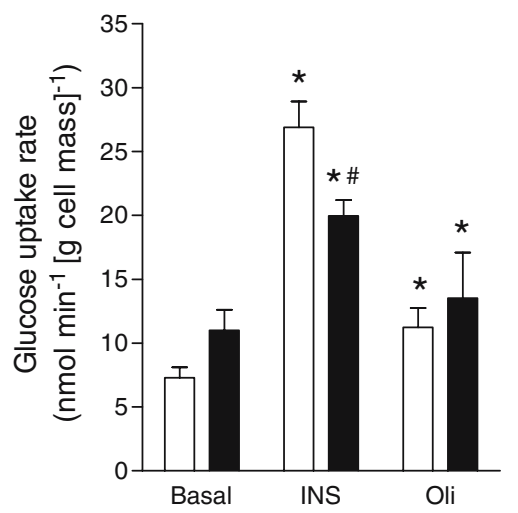

Fig. 2 Rates of 2-deoxyglucose uptake in cardiomyocytes from rats fed the LFD (open bars) and the HFD (filled bars). Cardiomyocytes were incubated with DMSO (Basal), insulin (INS) or oligomycin (Oli) before measurement of 2-deoxyglucose uptake. Data are expressed as wet cell mass and are means \pm SE; $n=8 .{ }^{*} p<0.02$, agonist effect; $\# p<$ 0.02 , diet effect 
a

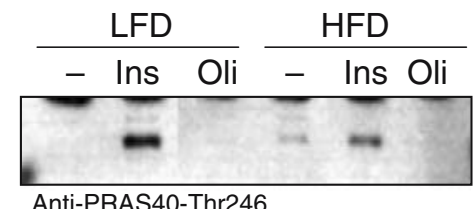

b

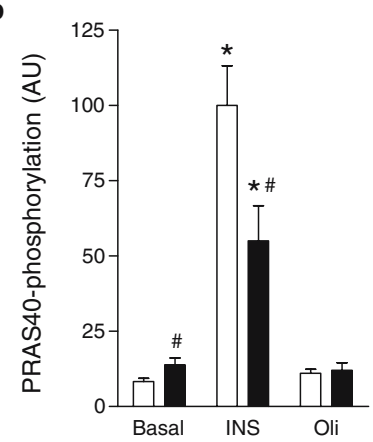

c
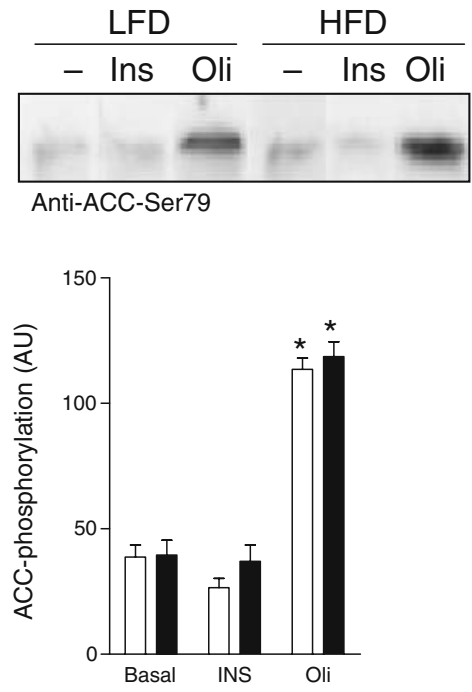

Fig. 3 Immunoblots (a, c) and quantification of PRAS40-Thr246 (b) and ACC-Ser79 (d) phosphorylation after incubation of cardiomyocytes from LFD- and HFD-fed rats with DMSO (Basal), insulin (INS) or oligomycin (Oli). Data are means $\pm \mathrm{SE} ; n=5$. ${ }^{*} p<0.05$, agonist effect; $\# p<0.05$, diet effect increased LCFA uptake in LFD and HFD cardiomyocytes to the same extent (Fig. 4; both $p<0.05$ vs basal).

We next examined the metabolic fate of LCFA following their uptake by HFD and LFD cardiomyocytes. Basal rates of LCFA oxidation were reduced by $22 \%$ in HFD relative to LFD cells (Fig. $4 \mathrm{~b} ; p<0.01$ ). SSO further lowered cardiomyocyte LCFA oxidation rates by $66 \%(p<0.005)$ and $31 \%(p<0.05)$ in LFD and HFD cells, respectively, but absolute rates of LCFA oxidation were comparable in SSOtreated LFD and HFD cells.

The incorporation rate of LCFA into triacylglycerol was increased 1.4-fold $(p<0.05)$ in HFD compared with LFD cells under basal conditions (Fig. 4c). SSO reduced triacylglycerol formation in both LFD and HFD cells (both $p<0.05$ vs basal). Notably, the absolute triacylglycerol esterification rates in SSO-treated cells did not differ between HFD- and LFD-fed rats. Also, the incorporation of LCFA into phospholipids under basal conditions was increased 1.5-fold in HFD vs LFD cells $(p<0.05$; Fig. $4 c)$, and was reduced by SSO in both LFD and HFD cells (both $p<0.05$ vs basal). Myocardial triacylglycerol content was $181 \pm 50 \mu \mathrm{g} / \mathrm{mg}$ protein in LFD rats and $330 \pm 114 \mu \mathrm{g} / \mathrm{mg}$ protein in HFD rats $(p<0.02)$ [2].

HFD induces cardiomyocyte hypertrophy and alters the subcellular localisation of CD36 The inhibitory effect of SSO suggests that the increased basal LCFA uptake and esterification rates in HFD cells can be ascribed to changes in the sarcolemmal pool of CD36. Western blot analysis indicated that the levels of CD36 were similar between HFD and LFD hearts (Fig. 5a). Immunohistochemical staining of cardiac LV tissue indicated cardiomyocyte hypertrophy, as reflected by an increased cross-sectional area ( $p<0.05$ vs LFD; Table 3), and showed a more abundant presence of CD36 at the sarcolemmal membrane,

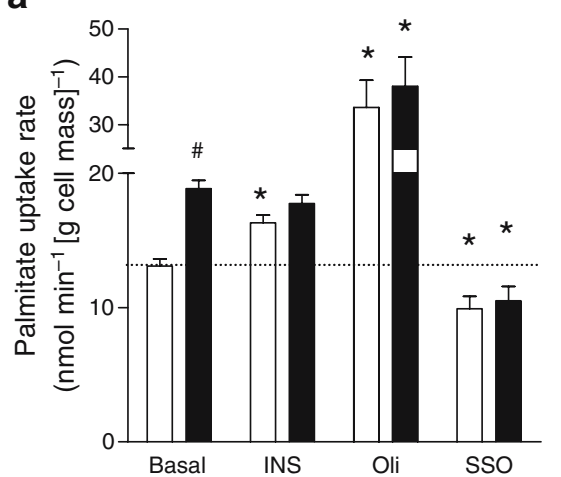

b

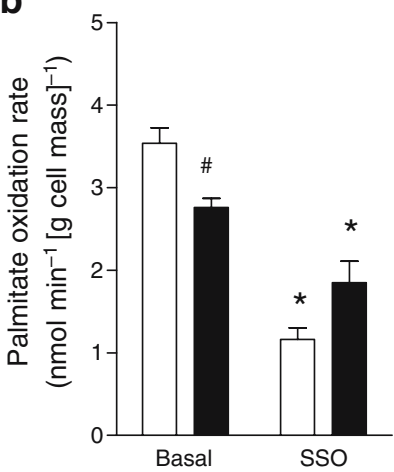

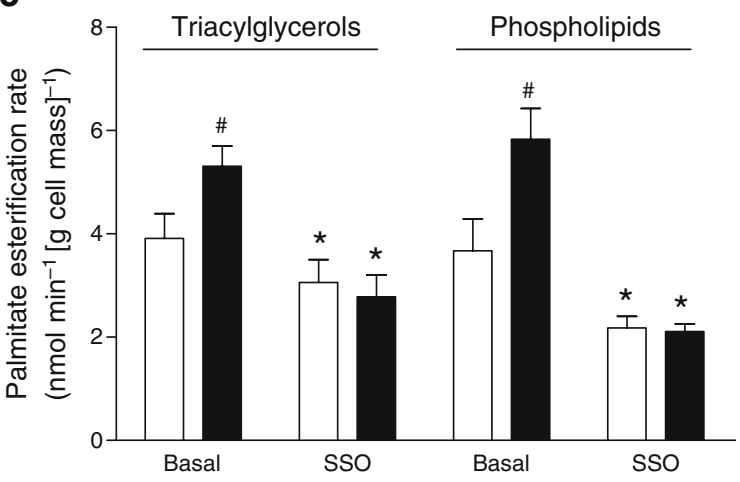

Fig. 4 Rates of palmitate uptake (a), oxidation (b) and esterification into intracellular triacylglycerols and phospholipids (c) in cardiomyocytes from LFD-fed rats (open bars) and HFD-fed rats (filled bars). Cardiomyocytes were incubated with DMSO (Basal), insulin (INS), oligomycin $(\mathrm{Oli})$ or SSO before measurement of palmitate uptake. Data are wet cell mass and are means \pm SE; $n=8 .{ }^{*} p<0.05$, agonist effect; $\# p<0.05$ diet effect 
particularly at the intercalated discs, in HFD hearts compared with LFD hearts ( $p<0.002$ vs LFD; Fig. 5b,c and Table 3).

As insulin stimulated LCFA uptake in isolated cardiomyocytes (Fig. 4), we also examined the subcellular localisation of CD36 in hearts isolated $30 \mathrm{~min}$ following an intraperitoneal injection with insulin (10 U/kg body weight) [2]. Figure $5 \mathrm{~d}$ shows that in vivo insulin treatment increased CD36 immunoreactivity at the intercalated discs in LFD hearts ( $p<0.01$ vs saline; Table 3 ). In HFD hearts, insulin did not further recruit CD36 to the sarcolemma (Fig. 5e).

HFD-induced CD36 redistribution to the sarcolemma precedes the onset of cardiac contractile dysfunction and associates with elevated basal phosphorylation of PKB/ $A k t$ We next analysed whether HFD-induced alterations in CD36 localisation precede the onset of cardiac contractile dysfunction, using rats fed an LFD or HFD for 4 weeks. This period was chosen based on serial echocardiographic measurements, which revealed a tendency towards decreased cardiac contractile function 4 weeks after initiation of the diet intervention. Specifically, FS\% was $4.2 \%$ lower $(p=0.07)$ and $\mathrm{EF} \%$ was $3.3 \%$ lower $(p=0.10)$ in HFD vs

\section{a}

LFD

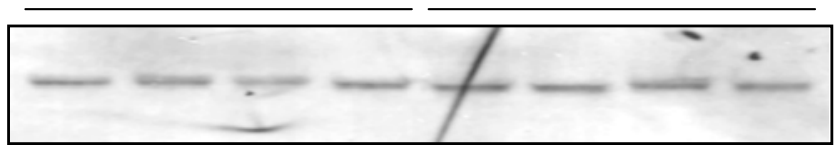

Anti-CD36

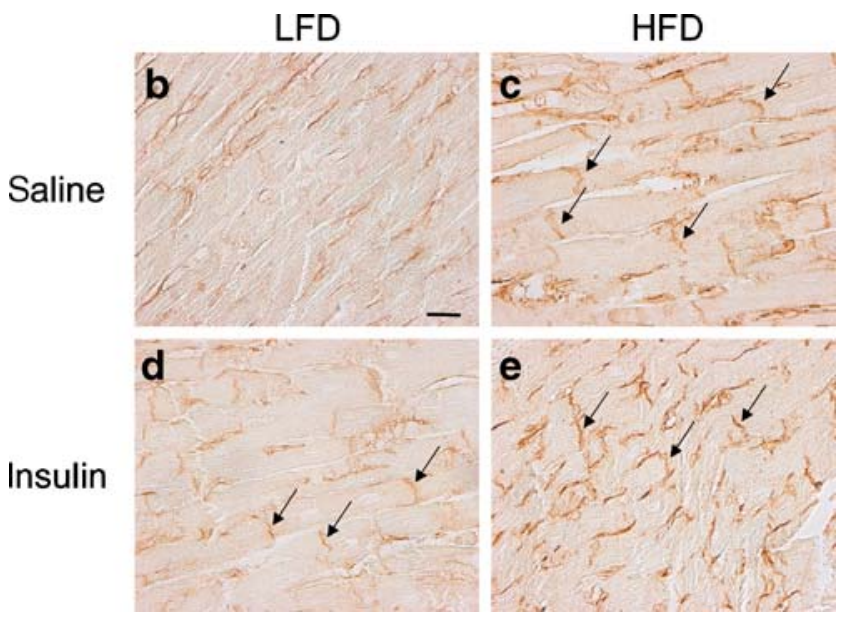

Fig. 5 Expression and subcellular localisation of CD36. Expression of CD36 in ventricular lysates of HFD and LFD rats. Ponceau S staining confirmed that the filters contained equal amounts of ventricular extracts (a). Immunohistochemical staining for CD36 localisation of cardiac LV tissue sections from rats fed an LFD $(\mathbf{b}, \mathbf{d})$ or HFD $(\mathbf{c}, \mathbf{e})$ for 8 weeks. Rats received an i.p. injection of saline $(\mathbf{b}, \mathbf{c})$ or insulin $(\mathbf{d}, \mathbf{e})$ $30 \mathrm{~min}$ before killing. Photographs are representative of two independent experiments performed on three rats per experimental group. Arrows indicate intercalated discs. Scale bar indicates $25 \mu \mathrm{m}$
LFD hearts after 4 weeks on the diet (data not shown), compared with the 9.2 and $6.2 \%$ decreases observed after 8 weeks of the feeding regime (both $p<0.05$; Table 1 ). Despite the absence of significant effects on cardiac function after 4 weeks of HFD feeding, immunohistochemical staining of cardiac LV tissue showed that the abundance of CD36 at the sarcolemmal membrane was already markedly increased (Fig. 6a,b) to levels almost similar to those observed after 8 weeks of HFD feeding (Fig. 5b,c).

Finally, we analysed whether HFD feeding induced changes in the activity of signalling pathways implicated in CD36 trafficking. In the healthy myocardium, CD36 is stored in at least two endosomal storage pools that are regulated by AMPK and PKB/Akt, respectively [26]. As insulin does not further recruit $\mathrm{CD} 36$ to the sarcolemma in HFD hearts (Fig. 5e), we proposed that increases in basal activity of $\mathrm{PKB} / \mathrm{Akt}$ may underlie the redistribution of CD36 in HFD hearts. Phosphorylation of PKB/Akt was determined in LV homogenates of the same group of salineinjected rats in which CD36 localisation was measured. As shown in Fig. 6c,d, basal phosphorylation of PKB/Akt was 1.7- and 2.6-fold higher after 4 and 8 weeks of HFD feeding, respectively (both $p<0.05$ ). Collectively, these results indicate that increased activity of $\mathrm{PKB} / \mathrm{Akt}$ may underlie CD36 redistribution to the sarcolemma, and that these events precede the LV dysfunction induced by HFD feeding.

\section{Discussion}

Here we report that HFD feeding induces cardiac contractile dysfunction in rats and that this is associated with a permanent relocation of $\mathrm{CD} 36$ to the sarcolemma. The continuous presence of CD36 at the sarcolemmal mem-

Table 3 Cardiac morphology and subcellular localisation of CD36

\begin{tabular}{|c|c|c|}
\hline & $\begin{array}{l}\text { LFD } \\
(n=6)\end{array}$ & $\begin{array}{l}\text { HFD } \\
(n=8)\end{array}$ \\
\hline \multicolumn{3}{|l|}{ Morphology } \\
\hline $\begin{array}{l}\text { Cardiomyocyte cross-sectional area } \\
\text { normalised to sarcomere length }\left(\mu \mathrm{m}^{2}\right)\end{array}$ & $214 \pm 3.5$ & $276 \pm 23 *$ \\
\hline \multicolumn{3}{|l|}{ CD36 localisation } \\
\hline \multicolumn{3}{|l|}{ Sarcolemma } \\
\hline Saline $(\%)$ & $61.7 \pm 3$ & $74.8 \pm 1 * *$ \\
\hline Insulin $(\%)$ & $75.9 \pm 4 * * *$ & $78.2 \pm 3$ \\
\hline \multicolumn{3}{|l|}{ Cytoplasm } \\
\hline Saline $(\%)$ & $38.3 \pm 3$ & $25.2 \pm 1 * *$ \\
\hline Insulin $(\%)$ & $24.1 \pm 4 * * *$ & $21.8 \pm 3$ \\
\hline
\end{tabular}

Data are means \pm SE

$* p<0.05$ vs LFD

$* * p<0.002$ vs LFD

$*_{* * *}<0.01$ vs saline 


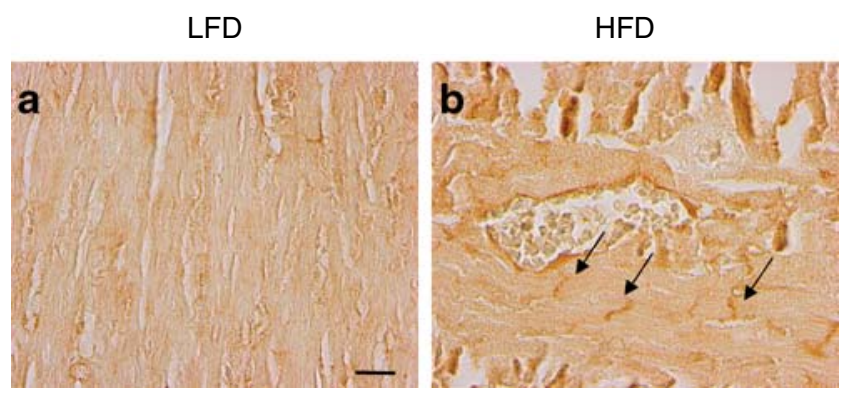

C

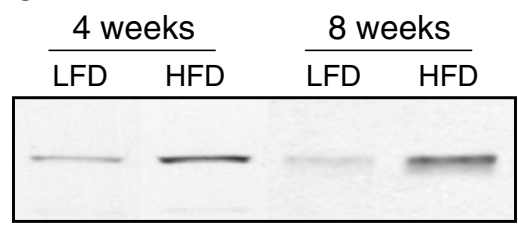

Anti-PKB/Akt-Ser473

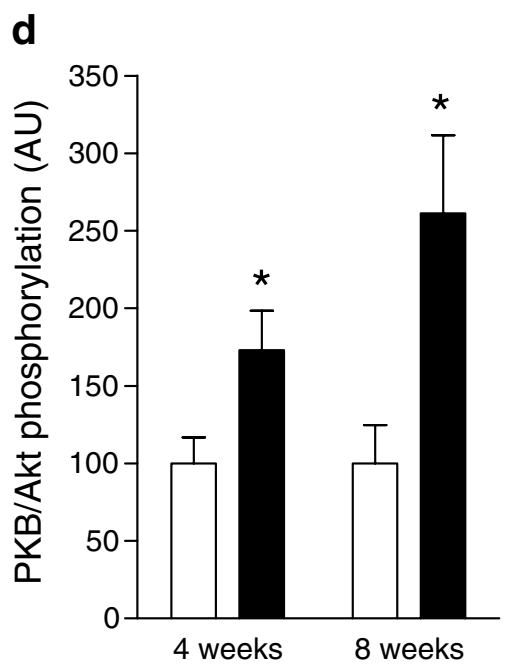

Fig. 6 Effect of the duration of HFD feeding on CD36 localisation and phosphorylation of PKB/Akt. Immunohistochemical staining for CD36 localisation of cardiac LV tissue sections from saline-injected rats fed an LFD (a) or HFD (b) for 4 weeks. Photographs are representative of two or three independent experiments performed on six rats per experimental group. The arrows indicate intercalated discs. The scale bar indicates $25 \mu \mathrm{m}$. $\mathbf{c}$ Immunoblot and quantification $\mathbf{d}$ of PKB/AktSer473 phosphorylation levels in ventricular homogenates from salineinjected rats fed an LFD (open bars) or HFD (filled bars) for 4 and 8 weeks, respectively. Data are means \pm SE, $n=8$. ${ }^{*} p<0.05$, diet effect

brane results in enhanced rates of LCFA uptake and subsequent esterification. We propose that this contributes to a decrease in myocardial insulin action and the development of diabetes-related heart disease. In addition, we show that AMPK-mediated responses are not affected by the composition of the diet.

A key observation in this study is that the alterations in cardiac contractile function in HFD hearts was associated with a continuous presence of CD36 at the sarcolemmal membrane. The present study provides the first morphological evidence for translocation of CD36 to the sarcolemmal membrane. Importantly, the amount of sarcolemmal CD36 closely correlated with enhanced LCFA uptake rates in isolated cardiomyocytes. The observation that SSO inhibited LCFA uptake in HFD cardiomyocytes to the same residual levels as measured in LFD cardiomyocytes provides further pharmacological evidence that the enhanced flux of LCFA in the heart of HFD-fed rats is a direct consequence of the relocalisation of CD36 to the sarcolemmal membrane.

Previously, a redistribution of CD36 to both subsarcolemmal and intramyofibrillar mitochondria has also been observed [27, 28]. Can the observed CD36 immunoreactivity be ascribed to subsarcolemmal mitochondria rather that the sarcolemma itself? Stimuli inducing translocation of CD36 to the mitochondria are expected not to discriminate between the subsarcolemmal and the intramyofibrillar mitochondria. Hence, if a stimulus or condition, in this case insulin or HFD feeding, were to increase the subsarcolemmal CD36 content, one would expect a similar increase in intramyofibrillar CD36 content. This, however, could not be confirmed in the immunohistochemical experiments. Furthermore, biochemical fractionations performed in previous studies substantiate the idea that a significant fraction of CD36 is present at the sarcolemma of hearts from insulin-resistant rats, and that only a minor portion of CD36 is found within the mitochondrial fractions $[9,22]$. While insulin has been shown to translocate CD36 to the sarcolemma [9, 22], insulin-mediated CD36 translocation to the mitochondria has never been reported and even seems counterintuitive. Collectively, the available evidence strongly supports the idea that the observed CD36 immunoreactivity can be ascribed to CD36 located at the sarcolemma rather than in subsarcolemmal mitochondria.

The combined data of this and our earlier study on obese Zucker rats [9] suggest that relocalisation of CD36 is a general phenomenon in insulin-resistant hearts, and raises the question of what mechanism underlies the continuous presence of CD36 at the sarcolemmal membrane in HFD hearts. In the healthy myocardium, CD36 is stored in at least two endosomal storage pools that are regulated by AMPK and PI3K/PKB/Akt, respectively [26]. Activation of AMPK is critical for contraction- and oligomycin-mediated CD36 translocation, whereas $\mathrm{PI} 3 \mathrm{~K} / \mathrm{PKB} / \mathrm{Akt}$ is critical for insulin-mediated CD36 trafficking [10, 14, 26]. Basal phosphorylation of PKB/Akt and its distal target, PRAS40, were elevated in HFD compared with LFD hearts, whereas AMPK activity was not affected by the diet. Furthermore, in cells from HFD-fed rats, the stimulatory effects of insulin on LCFA uptake, CD36 translocation and phosphorylation of PKB/Akt and PRAS40 were abrogated, whereas oligomycin- 
induced AMPK activation and LCFA uptake was unimpaired between cardiomyocytes from LFD- and HFD-fed rats. Although we cannot exclude a contribution of other kinases or HFD-induced alterations in the as yet undisclosed trafficking machinery regulating the internalisation of CD36 $[11,26]$, our observations do not argue against the suggestion that increases in $\mathrm{PKB} / \mathrm{Akt}$ activity may contribute to the sustained sarcolemmal presence of CD36 in the heart of HFD-fed rats.

Previously, we suggested that increased plasma insulin might contribute to $\mathrm{CD} 36$ relocalisation in hearts from obese Zucker rats [9]. As systemic hyperinsulinaemia was not observed in HFD-fed rats [2, 13], it remains interesting to examine whether changes in the activity of other (insulin-independent) regulators of PKB/Akt phosphorylation, such as PI3K $\gamma$ - and $\beta 2$-adrenergic receptor signalling pathways, $\mathrm{Ca}^{2+}$-calmodulin dependent kinase, protein phosphatase 2A and the sympathetic nervous system [29], can be linked to the observed increase in PKB/Akt phosphorylation in HFD hearts.

The metabolic and biochemical data were paralleled by in vivo cardiac functional changes. Importantly, HFDinduced cardiac contractile dysfunction does not seem to be linked to diet-related elevations in blood pressure. Circadian haemodynamic parameters were monitored using implanted telemetry devices in an experiment performed in a separate group of animals. We found a slight increase in night-time (activity-related) heart rate in HFD rats, but no changes in blood pressure even after 10 weeks of exposure to the diet (Electronic supplementary material Table 1). Others confirmed the absence of relevant hypertension after feeding rats a diet containing $74 \%$ fat for 17 weeks [30]. This underscores the possibility that alterations in metabolism, rather than in haemodynamics, underlie the impairment in cardiac contractile function in HFD rats.

The present study also suggests that HFD-induced alterations in the functional pool of CD36 may contribute to an imbalance in LCFA uptake and in oxidation and esterification rates in HFD vs LFD cells. Whereas LCFA uptake rates were increased 1.4-fold in HFD cells, the LCFA oxidation rates were not increased but rather modestly lower in HFD cells, while the rates of esterification into triacylglycerol and phospholipids were 1.4-fold higher in HFD vs LFD cardiomyocytes. Accordingly, triacylglycerol content was 1.9-fold increased in HFD compared with LFD hearts [2]. It seems unlikely that changes in malonylCoA levels underlie the reduced rates of LCFA oxidation in HFD hearts, as no diet-induced changes in ACC phosphorylation were found. Rather, degenerative changes in mitochondria, such as matrix dilution, cristolysis and mitochondria-associated lamellar bodies, have been observed in cardiomyocytes from HFD-fed rats [2] and have been linked to reduced oxidative capacity and lipid accumulation in skeletal muscle from patients with type 2 diabetes. However, further studies are required to examine whether these changes contribute to the reduced basal LCFA oxidation and increased LCFA esterification rates, or whether the extra LCFA taken up by HFD cells are stored as triacylglycerol and phospholipids as a consequence of HFD-induced changes in enzymes promoting LCFA esterification or inhibition of lipolysis.

An important finding of this study is that the HFDinduced CD36 redistribution preceded the onset of cardiac contractile dysfunction. Although we cannot unequivocally link CD36 redistribution to cardiac dysfunction, a recent report provided strong support for this notion as the absence of CD36 was found to prevent myocardial triacylglycerol accumulation in transgenic mice with cardiac PPAR $\alpha$ overexpression, both under normal conditions and after HFD feeding [12]. Similarly, alterations in PKB/Akt activity have been linked to cardiac dysfunction [31]. Myocardial biopsy samples obtained from patients with advanced heart failure or dilated cardiomyopathy show increased basal phosphorylation of PKB/Akt [32, 33], and transgenic mouse models with constitutively activated $\mathrm{PKB} / \mathrm{Akt}$ in the heart develop hypertrophy, decreased cardiac function and impaired recovery from ischaemiareperfusion injury $[31,34]$. Whereas chronic activation of $\mathrm{PKB} / \mathrm{Akt}$ increases basal glucose uptake rates in the heart [35], the effects on myocardial lipid metabolism have not been studied. Interestingly, PKB/Akt signalling has been linked to palmitate-induced beta cell lipotoxicity [36]. Based on this report and the findings described in this study, it would be of interest to analyse animals expressing chronically active $\mathrm{PKB} / \mathrm{Akt}$ for translocation of CD36 to the sarcolemma and myocardial lipid accumulation.

It seems plausible that an enhanced CD36-mediated LCFA uptake rate may contribute to the development of cardiac contractile dysfunction. An increased supply of LCFA may influence excitation-contraction coupling as well as other processes linked to cellular $\mathrm{Ca}^{2+}$ handling [37, 38]. Furthermore, triacylglycerol accumulation is strongly linked to cardiac insulin resistance and contractile dysfunction $[1,2,8]$. The dynamic equilibrium between triacylglycerol stores and triacylglycerol metabolites causes accumulation of ceramide and diacylglycerol during prolonged LCFA influx. Both ceramide and diacylglycerol have been implicated in the activation of serine/threonine kinases, such as PKC, Jun $\mathrm{N}$ terminal kinase (JNK), and inhibitor of nuclear factor-kB kinase (IKK), which counteract insulin signalling $[39,40]$. Notably, chronic myocardial activation of Akt in transgenic mice has also been linked to feedback inhibition of PI3K activity [33] and blunted insulin stimulation of glucose uptake [35]. Importantly, reduced insulin responsiveness reduces the ability of insulin to regulate substrate handling, e.g. metabolic inflexibility [41]. In this study and previous reports [2, 
13], we show that HFD feeding reduces insulin responsiveness and impairs metabolic flexibility, as illustrated by the blunted effects of insulin on the stimulation of glucose uptake, phosphorylation of PRAS40, LCFA uptake and translocation of CD36 to the sarcolemma.

We conclude that HFD feeding in rats induces cardiac contractile dysfunction, which is preceded by relocation of CD36 to the sarcolemma, and elevated basal levels of phosphorylated $\mathrm{PKB} / \mathrm{Akt}$. The continuous presence of CD36 at the sarcolemma contributes to enhanced rates of fatty acid uptake, resulting in myocardial triacylglycerol accumulation and accompanying insulin resistance. Collectively, these data suggest that alterations in the subcellular localisation of CD36 may contribute to the development of diabetes-related heart disease and that $\mathrm{CD} 36$ may be a therapeutic target to prevent cardiac dysfunction and the development of heart failure in diabetes.

Acknowledgements The authors acknowledge the support of the Dutch Diabetes Research Foundation (grants 2003.00.029 and 2004.00.052), the Netherlands Heart Foundation (NHF grants 2000.156 and 2002.T049), the Netherlands Organisation for Health Research and Development (NWO ZonMw grant 40-00812-9803075), and the European Commission (Integrated Project LSHMCT-2004-005272, Exgenesis). JFCG is the NHF Professor of Cardiac Metabolism. JJFPL is the recipient of a VIDI-Innovational Research Grant from the Netherlands Organization of Scientific Research (NWO-ZonMW Grant 016.036.305).

Duality of interest The authors declare that there is no duality of interest associated with this manuscript.

\section{References}

1. Fang ZY, Prins JB, Marwick TH (2004) Diabetic cardiomyopathy: evidence, mechanisms, and therapeutic implications. Endocr Rev 25:543-567

2. Ouwens DM, Boer C, Fodor M et al (2005) Cardiac dysfunction induced by high-fat diet is associated with altered myocardial insulin signalling in rats. Diabetologia 48:1229-1237

3. Zhou YT, Grayburn P, Karim A et al (2000) Lipotoxic heart disease in obese rats: implications for human obesity. Proc Natl Acad Sci USA 97:1784-1789

4. Belke DD, Larsen TS, Gibbs EM, Severson DL (2000) Altered metabolism causes cardiac dysfunction in perfused hearts from diabetic $(\mathrm{db} / \mathrm{db})$ mice. Am J Physiol Endocrinol Metab 279: E1104-E1113

5. Christoffersen C, Bollano E, Lindegaard ML et al (2003) Cardiac lipid accumulation associated with diastolic dysfunction in obese mice. Endocrinology 144:3483-3490

6. Mazumder PK, O'Neill BT, Roberts MW et al (2004) Impaired cardiac efficiency and increased fatty acid oxidation in insulinresistant ob/ob mouse hearts. Diabetes 53:2366-2374

7. Finck BN, Lehman JJ, Leone TC et al (2002) The cardiac phenotype induced by PPARalpha overexpression mimics that caused by diabetes mellitus. J Clin Invest 109:121-130

8. Taegtmeyer H, McNulty P, Young ME (2002) Adaptation and maladaptation of the heart in diabetes: Part I: general concepts. Circulation 105:1727-1733
9. Coort SL, Hasselbaink DM, Koonen DP et al (2004) Enhanced sarcolemmal FAT/CD36 content and triacylglycerol storage in cardiac myocytes from obese Zucker rats. Diabetes 53:16551663

10. Chabowski A, Coort SL, Calles-Escandon J et al (2005) The subcellular compartmentation of fatty acid transporters is regulated differently by insulin and by AICAR. FEBS Lett 579:2428-2432

11. Koonen DP, Glatz JF, Bonen A, Luiken JJ (2005) Long-chain fatty acid uptake and FAT/CD36 translocation in heart and skeletal muscle. Biochim Biophys Acta 1736:163-180

12. Yang J, Sambandam N, Han X et al (2007) CD36 Deficiency rescues lipotoxic cardiomyopathy. Circ Res 100:1208-1217

13. Nascimento EB, Fodor M, van der Zon GC et al (2006) Insulinmediated phosphorylation of the proline-rich Akt substrate PRAS40 is impaired in insulin target tissues of high-fat diet-fed rats. Diabetes 55:3221-3228

14. Luiken JJ, Coort SL, Willems J et al (2003) Contraction-induced fatty acid translocase/CD36 translocation in rat cardiac myocytes is mediated through AMP-activated protein kinase signaling. Diabetes 52:1627-1634

15. Luiken JJ, van Nieuwenhoven FA, America G, van der Vusse GJ, Glatz JF (1997) Uptake and metabolism of palmitate by isolated cardiac myocytes from adult rats: involvement of sarcolemmal proteins. J Lipid Res 38:745-758

16. van den Bos EJ, Mees BM, de Waard MC, de Crom R, Duncker DJ (2005) A novel model of cryoinjury-induced myocardial infarction in the mouse: a comparison with coronary artery ligation. Am J Physiol Heart Circ Physiol 289:H1291-H1300

17. Vasanji Z, Cantor EJ, Juric D, Moyen M, Netticadan T (2006) Alterations in cardiac contractile performance and sarcoplasmic reticulum function in sucrose-fed rats is associated with insulin resistance. Am J Physiol Cell Physiol 291:C772-C780

18. Slama M, Ahn J, Peltier M et al (2005) Validation of echocardiographic and Doppler indexes of left ventricular relaxation in adult hypertensive and normotensive rats. Am J Physiol Heart Circ Physiol 289:H1131-H1136

19. Fischer Y, Rose H, Kammermeier H (1991) Highly insulinresponsive isolated rat heart muscle cells yielded by a modified isolation method. Life Sci 49:1679-1688

20. Habets DD, Coumans WA, Voshol PJ et al (2007) AMPKmediated increase in myocardial long-chain fatty acid uptake critically depends on sarcolemmal CD36. Biochem Biophys Res Commun 355:204-210

21. Coort SL, Willems J, Coumans WA et al (2002) Sulfo- $N$ succinimidyl esters of long chain fatty acids specifically inhibit fatty acid translocase (FAT/CD36)-mediated cellular fatty acid uptake. Mol Cell Biochem 239:213-219

22. Luiken JJ, Koonen DP, Willems J et al (2002) Insulin stimulates long-chain fatty acid utilization by rat cardiac myocytes through cellular redistribution of FAT/CD36. Diabetes 51:3113-3119

23. Keizer HA, Schaart G, Tandon NN, Glatz JF, Luiken JJ (2004) Subcellular immunolocalisation of fatty acid translocase (FAT)/ CD36 in human type-1 and type-2 skeletal muscle fibres. Histochem Cell Biol 121:101-107

24. Beek-Harmsen BJ, van der Laarse WJ (2005) Immunohistochemical determination of cytosolic cytochrome $\mathrm{C}$ concentration in cardiomyocytes. J Histochem Cytochem 53:803-807

25. Zarrinpashneh E, Carjaval K, Beauloye C et al (2006) Role of the alpha2-isoform of AMP-activated protein kinase in the metabolic response of the heart to no-flow ischemia. Am J Physiol Heart Circ Physiol 291:H2875-H2883

26. Luiken JJ, Coort SL, Koonen DP, Bonen A, Glatz JF (2004) Signalling components involved in contraction-inducible substrate uptake into cardiac myocytes. Proc Nutr Soc 63:251-258

27. Holloway GP, Bezaire V, Heigenhauser GJ et al (2006) Mitochondrial long chain fatty acid oxidation, fatty acid translocase/CD36 
content and carnitine palmitoyltransferase I activity in human skeletal muscle during aerobic exercise. J Physiol 571:201-210

28. Schenk S, Horowitz JF (2006) Coimmunoprecipitation of FAT/ CD36 and CPT I in skeletal muscle increases proportionally with fat oxidation after endurance exercise training. Am J Physiol Endocrinol Metab 291:E254-E260

29. Poornima IG, Parikh P, Shannon RP (2006) Diabetic cardiomyopathy: the search for a unifying hypothesis. Circ Res 98:596-605

30. Fitzgerald SM, Henegar JR, Brands MW, Henegar LK, Hall JE (2001) Cardiovascular and renal responses to a high-fat diet in Osborne-Mendel rats. Am J Physiol Regul Integr Comp Physiol 281:R547-R552

31. O'Neill BT, Abel ED (2005) Akt1 in the cardiovascular system: friend or foe? J Clin Invest 115:2059-2064

32. Haq S, Choukroun G, Lim H et al (2001) Differential activation of signal transduction pathways in human hearts with hypertrophy vs advanced heart failure. Circulation 103:670-677

33. Nagoshi T, Matsui T, Aoyama $\mathrm{T}$ et al (2005) PI3K rescues the detrimental effects of chronic Akt activation in the heart during ischemia/reperfusion injury. J Clin Invest 115:2128-2138

34. Shiojima I, Walsh K (2006) Regulation of cardiac growth and coronary angiogenesis by the Akt/PKB signaling pathway. Genes Dev 20:3347-3365
35. Matsui T, Nagoshi T, Hong EG et al (2006) Effects of chronic Akt activation on glucose uptake in the heart. Am J Physiol Endocrinol Metab 290:E789-E797

36. Higa M, Shimabukuro M, Shimajiri Y, Takasu N, Shinjyo T, Inaba $T$ (2006) Protein kinase B/Akt signalling is required for palmitate-induced beta-cell lipotoxicity. Diabetes Obes Metab 8:228-233

37. Philipson KD, Ward R (1985) Effects of fatty acids on $\mathrm{Na}^{+}-\mathrm{Ca} 2^{+}$ exchange and $\mathrm{Ca}_{2}{ }^{+}$permeability of cardiac sarcolemmal vesicles. J Biol Chem 260:9666-9671

38. Huang JM, Xian H, Bacaner M (1992) Long-chain fatty acids activate calcium channels in ventricular myocytes. Proc Natl Acad Sci USA 89:6452-6456

39. Hotamisligil GS (2005) Role of endoplasmic reticulum stress and c-Jun NH2-terminal kinase pathways in inflammation and origin of obesity and diabetes. Diabetes 54(Suppl 2):S73-S78

40. Perseghin G, Petersen K, Shulman GI (2003) Cellular mechanism of insulin resistance: potential links with inflammation. Int J Obes Relat Metab Disord 27(Suppl 3):S6-S11

41. Taegtmeyer H, Golfman L, Sharma S, Razeghi P, van Arsdall M (2004) Linking gene expression to function: metabolic flexibility in the normal and diseased heart. Ann N Y Acad Sci 1015:202213 